\title{
Neural substrates of anorexia nervosa patient's deficits to decode emotional information
}

\author{
Dorothée Lulé $^{1}$ - Sabine Müller ${ }^{2}$ - Anne-Katharina Fladung ${ }^{3}$ - Ingo Uttner ${ }^{1}$ - Ulrike M. E . Schulze $e^{2,4}$
}

Received: 1 December 2019 / Accepted: 7 April 2020 / Published online: 1 May 2020

(c) The Author(s) 2020

\begin{abstract}
Purpose The aim of the study was to define specific substrates of pathological behaviour patterns by analysing cortical activity using functional magnetic resonance imaging (fMRI) during an emotional processing task.

Methods In a sample of $N=11$ adolescent patients with $\mathrm{AN}$ (16.36 years, $\mathrm{SD} \pm 1.36)$ and $N=11$ age-matched controls, we performed a functional MRI study to detect BOLD signal changes in a 3 T MRI scanner while presenting emotional facial stimuli.

Results Young people with AN presented with a generally reduced cortical activation pattern in key areas of emotion recognition for happy and fearful faces. Areas essential for control of social behaviour were associated with symptoms of depression. Conclusion Obviously, there are already indications of cortical patterns in young affected persons, which indicate a changed emotional reaction to potentially aversive stimuli in the sense of a changed top-down process of emotion avoidance. Thus, the current study provides further evidence that the disorder of anorexia nervosa is closely related to deficits in emotion processing in the early course of ontogenesis. Depressive symptoms might additionally trigger pathological behavior. Due to the small sample size, the data should be considered preliminary and require further validation.
\end{abstract}

Level of evidence Level of evidence III: case-control study.

Keywords Anorexia nervosa $\cdot$ Emotion recognition $\cdot$ Anxiety $\cdot$ Depression $\cdot$ Alexithymia

\section{Introduction}

The avoidance of both, the experience and expression of strong emotions, is typically observed in individuals with anorexia nervosa, and researchers claim that it is an important issue in the emergence and maintenance of the disease [1]. Accordingly, individuals with AN seem to show altered

Ulrike M. E . Schulze

ulrike.schulze@uniklinik-ulm.de

1 Department of Neurology, University of Ulm, Oberer Eselsberg 45, 89081 Ulm, Germany

2 Department of Child and Adolescent Psychiatry/Psychotherapy, University of Ulm, Steinhövelstrasse 5, 89075 Ulm, Germany

3 Institute of Psychology, Clinical Psychology and Psychotherapy, University of Hamburg, von-Melle-Park 5, 20146 Hamburg, Germany

4 Department of Child and Adolescent Psychiatry, Centre for Psychiatry Calw, Bunsenstraße 120, 71032 Böblingen, Germany emotional processing of visually presented faces and report higher alexithymia (i.e., a lower capability to identify and express their emotions) [2] than healthy individuals. Despite the probably crucial role of aberrant emotion processing in anorexia nervosa, research on its neural pathways is sparse: to our knowledge, only three fMRI studies on facial emotion perception in AN patients have been published so far, all of them examining adult subjects [3]. Findings are controversial, but overall indicating that neural correlates of facial emotion perception might be different in AN patients compared to healthy controls. To date, no fMRI studies on neural correlates of facial emotion perception in adolescents have been published.

In a previous study, we provided evidence that adolescents with AN present with recognition deficits of aversive stimuli such as disgust, whereas the positive emotion of happiness in faces was more easily recognized [4]. Emotion recognition deficits were with psychopathology, e.g., depressive symptoms. The aim of our current study was to determine cortical substrates of these emotion processing 
deficits in the previously reported adolescent sample [4] as evidence for early changes in anorexia nervosa.

\section{Materials and methods}

\section{Participants}

In total, $N=15$ female adolescents between 14 and 18 years diagnosed with AN participated in the study (for further details see [4]). They were recruited through the Department of Child and Adolescent Psychiatry/Psychotherapy of Ulm University. In addition, 15 female healthy comparison subjects, who were group-matched for age, handedness, and education level, were recruited in state schools in Ulm. For all participants, prerequisite was a stable physical condition that allowed them to take part in such a challenging study. Exclusion criteria were: suicidal tendencies, substance abuse and/or dependence, cardiovascular diseases, epilepsy, Tourette syndrome, brain diseases, and endocrine diseases (e.g., hyperthyroidism). Additional exclusion criteria for healthy controls (HC) were eating and other psychiatric disorders. Due to missing data, four participants from each group were excluded from analysis, respectively, leaving $N=11 \mathrm{AN}$ patients for the final analysis.

\section{Measures}

\section{General assessment}

All patients received a comprehensive clinical examination including medical and family history, neurological and psychiatric assessments and routine blood analysis to exclude physical illnesses apart from AN as reported previously (Table 1) [4]. Following clinical examination, participants performed fMRI scanning, followed by the FEEL test outside the MRI scanner [see 4] to determine subject's ability to recognize facially expressed emotions as previously described (Table 1) [4]. To minimize influences of circadian rhythm, patients and controls were asked to perform the tests at the same daytime.
Table 1 Demographics and psychopathology of AN patients compared to controls

\begin{tabular}{|c|c|c|c|c|c|c|}
\hline & \multicolumn{2}{|c|}{ Anorexia patients } & \multicolumn{2}{|c|}{ Healthy controls } & \multicolumn{2}{|l|}{$\mathrm{t}$ test } \\
\hline & Mean & SD & Mean & SD & $T / U / F$ & $P$ \\
\hline Age & 16.4 & 1.4 & 16.5 & 0.9 & (F) 0.3 & 0.62 \\
\hline BMI & 17.6 & 0.9 & 21.4 & 1.7 & $(F) 46.5$ & $<0.001 * *$ \\
\hline HAWIK & 102.3 & 9.4 & 101.6 & 5.8 & (T) 0.2 & 0.83 \\
\hline TAS & 49.7 & 13.6 & 39.7 & 7.4 & (T) 2.1 & $0.05 *$ \\
\hline EDI & 20.1 & 12.6 & 3.9 & 2.9 & $(U) 17.0$ & $0.003 * *$ \\
\hline EDE-Q & 2.5 & 1.94 & 0.2 & 0.2 & (T) 4.0 & $0.004 * *$ \\
\hline STAI state & 44.5 & 14.03 & 31.8 & 7.0 & (T) 2.7 & $0.02 *$ \\
\hline STAI trait & 47.9 & 11.01 & 32.6 & 8.1 & (T) 3.7 & $0.001 * *$ \\
\hline BDI & 265.7 & 70.3 & 185.7 & 30.2 & (T) 4.2 & $0.002 * *$ \\
\hline FEEL total & 34.4 & 4.1 & 35.4 & 4.6 & $(T)-0.5$ & 0.61 \\
\hline FEEL time & 1238.8 & 355.6 & 1581.6 & 548.8 & $(U) 29.0$ & $0.04 *$ \\
\hline FEEL happiness & 7.0 & 0 & 6.8 & 0.4 & $(U) 49.5$ & 0.15 \\
\hline FEEL surprise & 5.8 & 1.25 & 6.3 & 1.1 & $(U) 45.5$ & 0.33 \\
\hline FEEL disgust & 4.8 & 1.6 & 5.9 & 1.3 & $(U) 36.0$ & 0.12 \\
\hline FEEL anger & 6.7 & 0.5 & 6.6 & 0.5 & $(U) 49.5$ & 0.48 \\
\hline FEEL sadness & 6.1 & 1.8 & 6.3 & 1.6 & $(U) 47.0$ & 0.40 \\
\hline FEEL fear & 4.6 & 1.5 & 4.2 & 2.1 & (T) 0.6 & 0.56 \\
\hline
\end{tabular}

A threshold of $* p<0.05$ and $* * p<0.001$ (two-tailed) was used for statistical interference

Eating disorder psychopathology was assessed by the Eating Disorder Questionnaire (EDE-Q) and the Eating Disorder Inventory (EDI 2) for adolescents and adults. Depression was inquired by means of the Beck Depression Inventory II (BDI II). Total score (maximum 42 points) and the average response time per stimulus in the whole test is given. The 42 points are calculated by multiplying the six basic emotions and a maximum score per emotion of seven points. In addition, it can be determined which emotion was confused with which other emotion

HAWIK Hamburg-Wechsler-Intelligenztest for children-third edition (HAWIK-III), TAS Toronto alexithymia scale, STAI State and trait anxiety, FEEL facial expression of emotions test 


\section{fMRI scanning}

A functional MRI was performed on a 3 T Siemens Magnetom Allegra (Siemens, Erlangen, Germany). T2*_ weighted images sensitive for Blood Oxygen Level Dependent (BOLD) contrasts were acquired every $2.14 \mathrm{~s}$ (repetition time, TR) with a voxel size of $3.6 \times 3.6 \times 3.2 \mathrm{~mm}^{3}$. The echo time (TE) was $32 \mathrm{~ms}$ and the flip angle (FA) was 90. Thirtysix slices were acquired in interleaved descending direction (Field of View $=200 \mathrm{~mm}$, slice thickness $=3.2 \mathrm{~mm}, 25 \%$ interslice gap). Each of the three sessions contained 198 image volumes, resulting in a scan time of 7 min $8 \mathrm{~s}$ per session. The transversal slices were oriented along the AC/ $\mathrm{PC}$ line. In addition, high-resolution $T 1$-weighted anatomical images of the brain $(\mathrm{TR}=2080 \mathrm{~ms}, \mathrm{TE}=3.93 \mathrm{~ms}, \mathrm{FA}=12$, voxel size $=1 \times 1 \times 1 \mathrm{~mm}^{3}$ ) were obtained.

\section{fMRI design}

An event-related design was applied to the paradigm presented during fMRI measurement. It was developed in order to get optimized signal-to-noise ratio. The stimuli are part of the original version of the Japanese and Caucasian Facial Expressions of Emotion stimulus set (JACFEE). In total, 191 pictures depicting 180 expressions of six emotions (happiness, sadness, anger, fear, surprise and disgust) of 60 individuals (half of them were of Caucasian and half of Japanese origin and gender distribution was even) were presented via video goggles during fMRI measurement [see 4]. Every individual was seen on three pictures, showing one emotion with an intensity of 60,80 or $100 \%$. Ten pictures of neutral faces were also included. One grey picture was generated as an average of all faces as a visual non-facial stimulus. Participants were presented 78 trials of pictures in total, divided into three runs of 26 trials of pictures each. One trial consisted of three different faces showing the same emotion with the same intensity, each shown for one second and divided by a fixation cross shown for one second. The fixation cross at the end of each trial appeared for a random time period (8-12 s). One trial took between 13 and $17 \mathrm{~s}$ in total. Nine trials of the six emotions and 12 trials of the neutral face and the grey picture appeared, respectively. The intensities of the depicted emotions were evenly distributed. The order of the stimulus presentation was pseudorandomized.

The instruction was to pay attention to all emotions displayed on faces. To control for attention, a photo of a woman whose face was not part of the item pool was presented at the beginning of each run, and participants were instructed to count how often she appeared in the following run. Following, a fixation cross appeared for a random time period, and the presentation of the stimuli began.

\section{Statistical analysis}

\section{Behavioral and clinical data}

Questionnaire and test data were analysed using the Statistical Package for Social Sciences-24 (IBM® SPSS version 24.0). Statistical Parametric Mapping- 12 (Functional Imaging Laboratory, Institute of Neurology, UCL, London, UK) was used to conduct fMRI data analysis, running with Matlab R2016a (Release 2011b, The MathWorks, Inc., Natick, Massachusetts, United States). Analyses of self-report and behavioral data have been presented previously [4].

\section{fMRI data}

The first five volumes of the functional run were discarded. Functional images were corrected for slice acquisition temporal onset differences. Subsequently, spatial realignment was performed on each fMRI data series by aligning all scans from each run with the first scan of the first run and aligning the images within runs with the mean of the images after the first realignment, in order to correct for residual interscan movement artifacts. Time series were normalized to the standard Montreal Neurological Institute $T 1$ template (voxel size: $3.6 \times 3.6 \times 3.2 \mathrm{~mm}^{3}$ ) and spatially smoothed using a Gaussian kernel with a Full Width at Half Maximum (FWHM) of $8 \mathrm{~mm}$. The tool ArtRepair was used for motion artifact correction: Outliers were selected by large variations in average global intensity or excessive scan-to-scan motion, and outlier volumes were interpolated.

For each subject, a first-level analysis was applied separately for each run, including the following condition types: all faces, disgust, happiness, surprise, anger, sadness, fear and neutral faces. Regressors were defined with onsets at the time of appearance of the corresponding event and convolved with the canonical hemodynamic response function. For the emotion conditions (disgust, happiness, surprise, anger, sadness, fear), the emotions' intensities $(60 \%, 80 \%$, $100 \%$ ) were included in the model. Six additional regressors were added in order to model the movement- and rotation parameters as confounds. For each subject, contrast images were calculated separately for each emotion condition compared with visual non-facial stimulus.

The individual contrast images were entered into separate second-level analysis to identify predictive voxels across subjects. Group activation maps were calculated using one sample $t$ tests. Group comparisons were performed via two sample $t$ tests. Regression was performed to test the null hypothesis of zero correlation between the series of scans and patient's depressiveness (BDI). 
Due to the explorative nature of the study and the small sample size, a cluster-wise corrected (50 voxels) with $p<0.05$ was performed. Activation peaks were assigned to brain regions using probabilistic cytoarchitectonic mapping with the help of the SPM toolbox Anatomy, and via the SPM toolbox WFU Pickatlas. The WFU Pickatlas was further used to assign peaks to Brodmann Areas.

\section{Results}

\section{Group differences in brain responses}

Compared to the $\mathrm{HC}$ group, patients with AN showed a significantly reduced activation of the left midcingulate cortex (MCC; BA 31) during the processing of faces. There was no brain area that showed stronger activation in the AN group than in the $\mathrm{HC}$ group.

In response to happy faces, patients with AN revealed a significantly reduced activation of the right fusiform gyrus and a significantly reduced activation centred in the right caudate nucleus, extending to the right thalamus compared to healthy controls.

In response to fearful faces patients with AN showed a significantly reduced activation in the superior medial gyrus (i.e., the medial part of the superior frontal gyrus), predominantly on the left side extending to both sides of the posterior medial frontal cortex (pMFC) compared to healthy controls. In addition, they exhibited significantly reduced activation in the right side of the inferior frontal gyrus (pars orbitalis, Table 2).

AN patients presented with no increased activation to any stimuli compared to controls. Whole brain analysis revealed no significant group difference to the contrasts disgust, surprise, anger, and sadness.

\section{Correlation of brain responses with comorbid psychopathology scores in AN}

BOLD response in the orbital gyrus to facial stimuli independent of emotional valence significantly correlated negatively with BDI scores (Table 2).

\section{Discussion}

Studies published during the past years suggest altered activation in regions related to the fronto-striatal and limbic circuits in anorexia nervosa, which are suspected to play an important role in the development and maintenance of the psychopathology of AN. Our data also suggest an overall altered cortical pattern of emotion processing in AN. During facial processing, AN patients presented with a reduced BOLD activity in middle cingulate gyrus which is a key area of information processing in unpleasant context and to control the negative impact of potentially aversive social
Table 2 MRI activation pattern in $\mathrm{AN}$ in response to faces with different emotional expressions

\begin{tabular}{|c|c|c|c|c|c|c|c|c|}
\hline & \multirow[t]{2}{*}{ Brain region } & \multirow[t]{2}{*}{ Side } & \multicolumn{3}{|c|}{ Peak MNI } & \multirow[t]{2}{*}{ K voxels } & \multirow[t]{2}{*}{$T(\mathrm{df})$} & \multirow[t]{2}{*}{$P$} \\
\hline & & & $\mathrm{x}$ & $\mathrm{y}$ & $\mathrm{Z}$ & & & \\
\hline \multicolumn{9}{|c|}{$\begin{array}{l}\text { Activation in response to faces with different emotional expressions in patients with anorexia nervosa and } \\
\text { healthy controls }\end{array}$} \\
\hline \multicolumn{9}{|l|}{ All faces } \\
\hline $\mathrm{HC}>\mathrm{AN}$ & Middle cingulate cortex (BA31) & $\mathrm{L}$ & -5 & -29 & 42 & 101 & $5.60(20)$ & $<0.001$ \\
\hline $\mathrm{HC}<\mathrm{AN}$ & None & & & & & & & \\
\hline \multicolumn{9}{|l|}{ Happy faces } \\
\hline \multirow[t]{2}{*}{$\mathrm{HC}>\mathrm{AN}$} & $\begin{array}{l}\text { Fusiform gyrus } \\
\text { (BA 37) }\end{array}$ & $\mathrm{R}$ & 31 & -44 & -12 & 50 & $5.46(20)$ & $<0.001$ \\
\hline & Caudate nucleus, thalamus & $\mathrm{R}$ & 17 & -4 & 16 & 51 & $4.88(20)$ & $<0.001$ \\
\hline $\mathrm{HC}<\mathrm{AN}$ & None & & & & & & & \\
\hline \multicolumn{9}{|c|}{ Fearful faces } \\
\hline \multirow[t]{2}{*}{$\mathrm{HC}>\mathrm{AN}$} & Superior medial gyrus & $\mathrm{L}$ & 2 & 32 & 48 & 71 & $4.81(20)$ & $<0.001$ \\
\hline & Inferior frontal gyrus & $\mathrm{R}$ & 38 & 28 & -6 & 71 & $4.53(29)$ & $<0.001$ \\
\hline $\mathrm{HC}<\mathrm{AN}$ & None & & & & & & & \\
\hline \multicolumn{9}{|c|}{ Correlation of BOLD response in fMRI with psychopathology in patients with anorexia nervosa } \\
\hline \multicolumn{9}{|l|}{ All faces } \\
\hline BDI $(-)$ & Orbital gyrus & $\mathrm{L}$ & -5 & 50 & -3 & 53 & 7.19 (13) & $<0.001$ \\
\hline
\end{tabular}

Threshold for significance uncorrected $p<0.001$ and cluster-wise uncorrected $p<0.05$ (50 voxels) $B A$ Brodman area, $M N I$ Montreal Neurological Institute, $A N$ patients with anorexia nervosa, $H C$ healthy controls, $B D I$ Beck Depression Inventory 
contexts [5]. For unpleasant stimuli such as fear, we found a significantly reduced cortical activity in superior medial frontal lobe, an area, which is crucial for emotion regulation and mentalizing [6]. This pattern of reduced cortical activity in areas of emotional processing is in line with the reduced capacity to recognize negative emotional face expressions [4]. However, participants displayed reduced performance for the facial emotion of disgust on the behavioral level in our previous study, whereas a subsample of this cohort which was measured in the current fMRI study showed no reduced cortical activity for disgust in any cerebral area which might be a matter of statistical power. Reduced cerebral activity was seen only for faces in general and for specific emotions of fear and happiness. The lack of activity in key areas of emotion recognition and regulation might suggest an additional top-down mechanism for fear and happiness in faces: the reduced activity in emotion processing and regulation areas might be facilitated by an avoidance strategy of subjects during a visual presentation of potentially (aversive) but also pleasant stimuli of facial expressions. However, as we did not provide any oculomotor data to determine fixation strategies of subjects (although all subjects were instructed to fixate the faces) and subject numbers were too low to correct for multiple comparisons, the discussion remains speculative.

Increased depression scores were associated with reduced orbitofrontal activity, an area, which is crucial for control of socially appropriate behaviour in specific, for appropriate food intake [7]. Orbitofrontal activity has been associated with the evolution of depression later in life [8]. The more depressed the patients in the current study were (as measured by BDI score), the less the area controlling appropriate behaviour such as food intake was activated. As loss of appetite and reduced food intake are key features of an affective state disorder, the pathologically reduced food intake in AN might additionally be triggered by this orbitofrontal loop via comorbid depressive symptoms.

In conclusion, adolescents with AN display an altered pattern of cortical BOLD activation. This pattern is suggestive of a top-down avoidance pattern of facial stimuli which are easily recognized. Our data are in agreement with previous research point to a significant involvement of the striatal reward system in AN. Altogether, we provide evidence for a multisystem disorder, with altered cortical pathways of social-emotional information processing of faces, which might be associated with avoidance strategies in social situations [9]. This complex interplay of dysfunctional activity in respective areas may lead to comorbid psychopathology, which then drives maladaptive behaviours [10] very early in development as we hereby present these changes in adolescent subjects.

\section{What is already known on this subject?}

Patients with anorexia nervosa often find it difficult-frequently in part due to comorbid symptoms such as alexithymia, depression and anxiety symptoms - to perceive and express their own (negative or strong) feelings. The often limited perception of the other person's emotions (via facial expressions) reinforces AN, especially as it can to contribute social withdrawal, a significant prognostic factor. So far, the neuronal correlates of the recognition of facial emotions have not been sufficiently researched-especially in the group of adolescents.

\section{What does this study add?}

Our results suggest a higher complexity in neuronal changes related to emotion processing in patients with anorexia nervosa than previously thought. However, based on the model of a "multisystem disorder", an etiological connection between perception, psychopathology and altered behaviour can be created. Further exploration of this connection would be desirable because it could lead to a holistic understanding of $\mathrm{AN}$ in young people.

Acknowledgements Open Access funding provided by Projekt DEAL. We would like to thank all patients and carers who supported us with their participation in the study. We thank Kathrin Bauer and Friederike Schöll for data acquisition and Fanni Dzvonyar for her support concerning fMRI data analysis. Special thanks to Prof. Harald Traue and his research team, Ulm University, for providing us with the JACFEE.

Funding This study was not (externally) funded.

\section{Compliance with ethical standards}

Conflict of interest UME Schulze received funding by the EU FP7 programme and actually serves an unpaid ethics advisor for two other EU-funded projects. She received speaker's fee from Shire. All other authors declare that they have no conflicts of interest. The authors alone are responsible for the content and writing of the paper.

Ethical approval All procedures performed in studies involving human participants were in accordance with the ethical standards of the institutional and/or national research committee (ethics committee of the University of Ulm, Germany; application no. 195/07) and with the 1964 Helsinki declaration and its later amendments or comparable ethical standards. Participants and their parents were informed about the procedures and potential risks of the study. Written informed consent and assent were obtained from all participants and their caregivers. Participants received a refund of a $30 €$ coupon.

Informed consent All patients and their caregivers gave their written (assent resp.) consent to participate in the study.

Open Access This article is licensed under a Creative Commons Attribution 4.0 International License, which permits use, sharing, adaptation, distribution and reproduction in any medium or format, as long as you give appropriate credit to the original author(s) and the source, 
provide a link to the Creative Commons licence, and indicate if changes were made. The images or other third party material in this article are included in the article's Creative Commons licence, unless indicated otherwise in a credit line to the material. If material is not included in the article's Creative Commons licence and your intended use is not permitted by statutory regulation or exceeds the permitted use, you will need to obtain permission directly from the copyright holder. To view a copy of this licence, visit http://creativecommons.org/licenses/by/4.0/.

\section{References}

1. Schmidt U, Treasure J (2006) Anorexia nervosa: valued and visible. A cognitive-interpersonal maintenance model and its implications for research and practice. Br J Clin Psychol 45(3):343-366. https://doi.org/10.1348/014466505X53902

2. Kessler H, Schwarze M, Filipic S, Traue HC, von Wietersheim J (2006) Alexithymia and facial emotion recognition in patients with eating disorders. Int J Eat Disord 39(3):245-251. https://doi. org/10.1002/eat.20228

3. Fonville L, Giampietro V, Surguladze S, Williams S, Tchanturia $\mathrm{K}$ (2014) Increased bold signal in the fusiform gyrus during implicit emotion processing in anorexia nervosa. NeuroImage Clin 4:266-273. https://doi.org/10.1016/j.nicl.2013.12.002

4. Lulé D, Schulze UM, Bauer K, Schöll F, Müller S, Fladung AK, Uttner I (2014) Anorexia nervosa and its relation to depression, anxiety, alexithymia and emotional processing deficits. Eat Weight Disord 19(2):209-216. https://doi.org/10.1007/s4051 9-014-0101-z

5. Pereira MG, de Oliveira L, Erthal FS, Joffily M, Mocaiber IF, Volchan E, Pessoa L (2010) Emotion affects action: midcingulate cortex as a pivotal node of interaction between negative emotion and motor signals. Cogn Affect Behav Neurosci 10(1):94-106. https://doi.org/10.3758/CABN.10.1.94

6. Schneider-Hassloff H, Straube B, Nuscheler B, Wemken G, Kircher T (2015) Adult attachment style modulates neural responses in a mentalizing task. Neuroscience 303:462-473. https ://doi.org/10.1016/j.neuroscience.2015.06.062

7. Frank MG, Stennett J (2001) The forced-choice paradigm and the perception of facial expressions of emotion. J Pers Soc Psychol 80(1):75-85. https://doi.org/10.1037//0022-3514.80.1.75

8. Jin J, Narayanan A, Perlman G, Luking K, DeLorenzo C, Hajcak G, Klein DN, Kotov R, Mohanty A (2017) Orbitofrontal cortex activity and connectivity predict future depression symptoms in adolescence. Biol Psychiatry Cogn Neurosci Neuroimaging 2(7):610-618

9. Wagner A, Aizenstein H, Venkatraman VK, Fudge J, May JC, Mazurkewicz L, Frank GK, Bailer UF, Fischer L, Nguyen V, Carter C, Putnam K, Kaye WH (2007) Altered reward processing in women recovered from anorexia nervosa. Am J Psychiatry 164:1842-1849. https://doi.org/10.1176/appi.ajp.2007.07040575

10. Lipsman N, Woodside DB, Lozano AM (2015) Neurocircuitry of limbic dysfunction in anorexia nervosa. Cortex 62:109-118. https ://doi.org/10.1016/j.cortex.2014.02.020

Publisher's Note Springer Nature remains neutral with regard to jurisdictional claims in published maps and institutional affiliations. 